\title{
Energy dependence of a low frequency QPO in GRS 1915+105
}

\author{
J. Rodriguez ${ }^{1}$, Ph. Durouchoux ${ }^{1}$, I. F. Mirabel ${ }^{1,2}$, Y. Ueda $^{3}$, M. Tagger ${ }^{1}$, and K. Yamaoka ${ }^{4}$ \\ 1 DSM/DAPNIA/Service d'Astrophysique (CNRS URA 2052), CEA Saclay, 91191 Gif-sur-Yvette, France \\ 2 Instituto de Astronomía y Física del Espacio/CONICET, Buenos Aires, Argentina \\ 3 Institute of Space and Astronautical Science, Yoshinodai 3-1-1, Sagamihara, Kanagawa 229-8510, Japan \\ 4 RIKEN The Institute of Physical and Chemical Research, Hirosawa 2-1, Wako, Saitama 351-0198, Japan
}

Received 26 October 2001 / Accepted 5 February 2002

\begin{abstract}
We analyze a set of three RXTE Target of Opportunity observations of the Galactic microquasar GRS 1915+105, observed on April 2000, during a multi-wavelength campaign. During the three observations, a strong, variable low frequency $(2-9 \mathrm{~Hz})$ quasi periodic oscillation (hereafter QPO), often referred to as the ubiquitous QPO, is detected together with its first harmonic. We study the spectral properties of both features, and show that: 1) their frequency variations are better correlated with the soft X-ray flux $(2-5 \mathrm{keV})$, favoring thus the location of the QPO in the accretion disk; 2) the QPO affects more the hard X-rays, usually taken as the signature of an inverse Compton scattering of the soft photons in a corona; 3) the fundamental and its harmonic do not behave in the same manner: the fundamental sees its power increase with the energy up to $40 \mathrm{keV}$, whereas the harmonic increases up to $\sim 10 \mathrm{keV}$. The results presented here could find an explanation in the context of the Accretion-Ejection Instability, which could appear as a rotating spiral or hot point located in the disk, between its innermost edge and the co-rotation radius. The presence of the harmonic could then be a signature of the non-linear behavior of the instability. The high-energy $(>40 \mathrm{keV})$ decrease of the fundamental would favor an interpretation where most or all of the quasi-periodic modulation at high energies comes not from the comptonized corona as usually assumed, but from a hot point in the optically thick disk.
\end{abstract}

Key words. stars: individual: GRS 1915+105 - X-rays: binaries

\section{Introduction}

$\mathrm{X}$-ray binaries exhibit strong X-ray emission, from the soft $(\sim 0.1 \mathrm{keV})$ to the hard X-rays (up to a few hundred keV), sometimes up to the $\mathrm{MeV}$ domain. The emission processes are thought to occur in the close vicinity of a stellarmass compact object (either a Neutron Star or a Black Hole), the soft part of the spectrum being usually taken as the thermal emission of an accretion disk, whereas the hard part is thought to be the manifestation of an inverse compton scattering of the soft photons, with relativistic electrons present in a hot coronal medium. The sources may be distinguished by several characteristics, such as the companion mass, whenever this latter is known, the shape of their spectra, or by the presence of strong collimated ejecta. In the latter case, the similarity with AGN led to the definition of microquasars (Mirabel et al. 1992), some of them known to be sources with superluminal jets (Mirabel \& Rodríguez 1999).

GRS 1915+105 has first been discovered as a Soft X-ray Transient by WATCH on board GRANAT (CastroTirado et al. 1992), and then identified as the first

Send offprint requests to: J. Rodriguez,

e-mail: rodrigue@discovery.saclay.cea.fr
Galactic source to have ejections with apparent superluminal motion (Mirabel \& Rodríguez 1994). The distance to the source has been estimated as $12.5 \mathrm{kpc}$, its inclination $\sim 70^{\circ}$, and the velocity of the jet $0.92 \mathrm{c}$ (Mirabel \& Rodríguez 1994). Since then, the source has been observed with many X-ray satellites, and its spectrum is typical of that of Black Hole Candidates (BHC), such as GRO J1655-40. Only recently, however, the spectral type of the companion has been identified as a $\mathrm{K}-\mathrm{M}$ III star (Greiner et al. 2001), classifying the source as a low-mass $\mathrm{X}$-ray binary. The mass of the primary has been estimated to $14 \pm 4 M_{\odot}$ (Greiner et al. 2001), confirming the black hole nature of the compact object.

With the launch of the Rossi X-ray Timing Experiment (RXTE), and the excellent timing capacities of both its pointed instruments, the Proportional Counter Array (PCA) and the High Energy X-ray Timing Experiment (HEXTE), many X-ray Binaries and GRS 1915+105 in particular, have been discovered to exhibit Quasi Periodic Oscillations (QPOs), in several ranges of frequency (a few $\mathrm{mHz}$ up to hundred, and kilohertz in the case of neutron star primary). Though no physical explanation has yet been widely accepted, the QPOs are thought to occur in the close vicinity of the compact object. 
Table 1. List of the Observations reduced; the interval time are those define by the PCA good time intervals as defined in Sect. 2. Relative time zero corresponds to $12 \mathrm{~h} 52 \mathrm{~m} 15 \mathrm{~s}$, start of the good time interval for interval \#1.

\begin{tabular}{|c|c|c|c|c|c|c|}
\hline Date & MJD & Obs Id & Interval \# & Time start (UT) & Time stop (UT) & PCUs "On" \\
\hline \multirow[t]{2}{*}{04172000} & 51651 & $50405-01-01-00$ & 1 & $12 \mathrm{~h} 52 \mathrm{~m} 15 \mathrm{~s}$ & $13 \mathrm{~h} 42 \mathrm{~m} 55 \mathrm{~s}$ & $0-4$ \\
\hline & & & 2 & $14 \mathrm{~h} 27 \mathrm{~m} 43 \mathrm{~s}$ & $15 \mathrm{~h} 18 \mathrm{~m} \mathrm{39s}$ & $0-4$ \\
\hline \multirow[t]{3}{*}{04222000} & 51656 & $50405-01-02-00$ & 1 & $09 \mathrm{~h} 21 \mathrm{~m} \mathrm{35s}$ & $10 \mathrm{~h} 15 \mathrm{~m} 27 \mathrm{~s}$ & $0-4$ \\
\hline & & $50405-01-02-01$ & 2 & $10 \mathrm{~h} 55 \mathrm{~m} 59 \mathrm{~s}$ & $11 \mathrm{~h} 38 \mathrm{~m} \mathrm{07s}$ & $0,2-4$ \\
\hline & & $50405-01-02-02$ & 3 & $12 \mathrm{~h} 31 \mathrm{~m} 59 \mathrm{~s}$ & $13 \mathrm{~h} 14 \mathrm{~m} 07 \mathrm{~s}$ & $0,2-4$ \\
\hline \multirow[t]{2}{*}{04232000} & 51657 & $50405-01-03-00$ & 1 & $07 \mathrm{~h} 40 \mathrm{~m} \mathrm{31s}$ & $08 \mathrm{~h} 35 \mathrm{~m} \mathrm{27s}$ & $0,2,3$ \\
\hline & & & 2 & $09 \mathrm{~h} 16 \mathrm{~m} 15 \mathrm{~s}$ & $09 \mathrm{~h} 59 \mathrm{~m} 59 \mathrm{~s}$ & $0,2,3$ \\
\hline
\end{tabular}

Furthermore, it has been pointed out by Psaltis et al. (1999) that the QPOs could represent the same type of variability in both neutron stars and black hole systems, constraining the theoretical models, and giving important clues to the physics of these phenomena. In particular the study of QPOs should give important information on the accretion flow, and thus on the physics of the disk.

The detection of several types of QPOs can be attributed to different mechanisms, depending in particular on the source spectral state.

We will only focus here on the strong $\sim 0.5-10 \mathrm{~Hz}$ QPO, present during the low/hard spectral state of GRS 1915+105, often called "ubiquitous", since it is nearly always present in that state and often observed in other Black Hole Binaries (e.g. XTE J1550-564, or GRO J1655-40). In that case, several authors have pointed out correlations between the frequency of the oscillations and some of the spectral parameters, such as the flux (Swank et al. 1997; Markwardt et al. 1999), the temperature of the disk (Muno et al. 1999), and the disk color radius (Rodriguez et al. 2002).

All these correlations constrained the location of the QPO in or close to the disk, and the systematic study of the QPO parameters should lead to a better understanding of the accretion and ejection mechanisms, thought to occur in this region.

Recently a new mechanism has been proposed by Tagger \& Pellat (1999), to extract energy and angular momentum from the inner regions of the disk (permitting, thus the accretion) and transport them toward the corotation radius of the spiral wave formed in the disk, where they can be emitted directly toward the corona (Tagger \& Pellat 1999; Varnière \& Tagger 2001).

It has been shown by Rodriguez et al. (2001), and Varnière et al. (2002), that this model could explain the different frequency vs. radius correlations observed in GRO J1655-40 compared to GRS $1915+105$ or (as had been found by Sobczak et al. 2000) XTE J1550-564.

This model could also explain the correlations found by Mirabel et al. (1998), Eikenberry et al. (1998), Ueda et al. (2002, our observations being part of this latter work) during the $\sim 30$ min cycle (Tagger 1999 for a possible scenario), between X-ray light curves and the infrared and radio emissions, considered as the synchrotron signatures of an expanding ejected blob of material, relating then the energy needed to accelerate those blobs to the one extracted from the accretion.

We present here observations of the source taken as a RXTE Target of Opportunity, in April 2000. In Sect. 2 we present the data reduction and analysis methods used; in Sect. 3, we examine the first of the three observations, which is the most variable one, and focus then on the dynamical properties of the source, observed in different energy ranges. In Sect. 4 we study the data of the following observations, where the source is much more steady, and thus, more adapted to extract the QPO parameters with high accuracy; we will interpret our observations in the last part of this paper.

\section{Data reduction and analysis}

The source has been observed on April 17th, 22nd and 23rd, 2000 as a target of opportunity. We have reduced and analyzed the processed data using the FTOOLS package (update 5.04). Observations IDs, exact time intervals, and dates are shown in Table 1.

We first extracted, for the three observations, lightcurves covering the entire PCA energy range, from binned data with $2^{-7} \mathrm{~s}=7.8125 \mathrm{~ms}$ resolution, and event data with $2^{-16} \mathrm{~s}=15.25878 \mu \mathrm{s}$, which were rebinned during the extraction process to $7.8125 \mathrm{~ms}$.

In all cases, lightcurves were extracted from all the PCUs that were simultaneously turned "on" over a single interval (i.e. 5 on Apr. 17th, and 22nd first interval, four during the two following intervals that day, and three on Apr. 23rd). We combined all PCUs and all layers to get the most possible incoming flux. The exact PCA configuration over each interval is given in Table 1.

"Good Time Intervals" (GTIs) were defined when the elevation angle was above $10^{\circ}$, the offset pointing less than $0.02^{\circ}$, and we also excluded the data taken while crossing the SAA.

Background lightcurves were generated using the PCABACKEST tool, from standard2 data, and subtracted from the raw lightcurves. We then generated power spectra and dynamical power spectra (hereafter DPS) using POWSPEC 1.0, calculating each FFT over $\sim 4 \mathrm{~s}$ time intervals (2048 bins in each intervals), and averaging then 


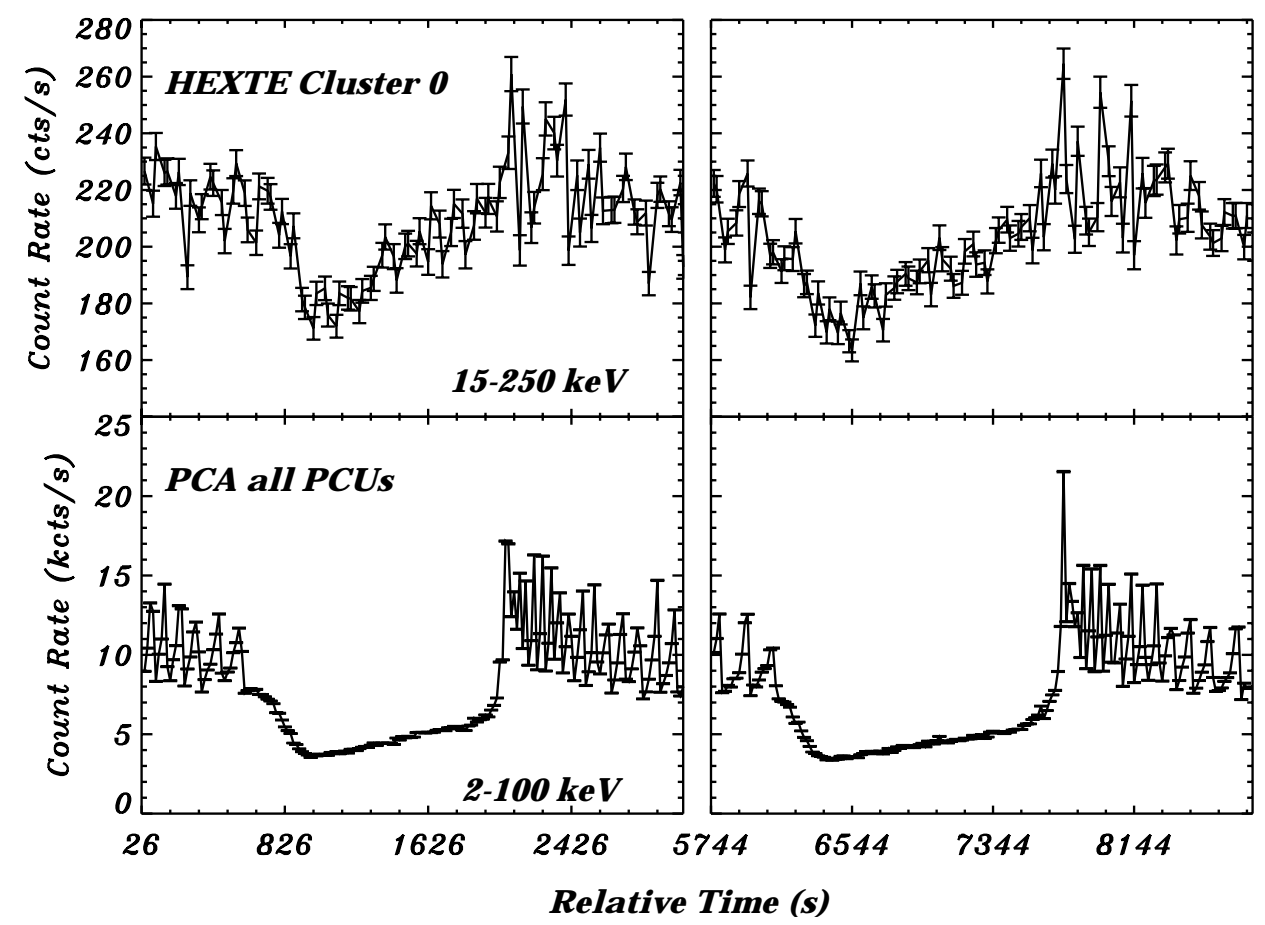

Fig. 1. The source on April 17th. Standard lightcurves covering in both cases the entire instrument energy range $(\sim 15-250$ keV for HEXTE, and $\sim 2-100 \mathrm{keV}$ for PCA); Upper panel: HEXTE Cluster 0 standard lightcurves with $16 \mathrm{~s}$ time bins, Lower panel: PCA standard 2 lightcurves with $16 \mathrm{~s}$ time bins. $X$ axis is in unit of s; $Y$ axis is in unit of cts/s (upper panels), and kcts/s (lower ones). Error bars are $1 \sigma$ statistical errors.

the result over 4 intervals. The resultant DPS has a resultant time bin $\sim 16 \mathrm{~s}$, comparable to the time resolution of the standard 2 lightcurves. To follow the evolution of the QPOs parameters with the energy, we extracted, in the same standard way, lightcurves in five PCA energy channels: absolute channel 0-11 (in Matrix epoch4 corresponding to $<2-4.99 \mathrm{keV})$, channel 12-29 (4.99-12.68 keV), channel 30-46 (12.68-20.06 keV), channel 47-89 (20.06$39.29 \mathrm{keV})$, channel 90-174 (39.29-80.04 keV). We then produced DPS and power spectra, as explained above, in each energy range.

\section{First observation: on April 17th}

We extracted from both instruments standard lightcurves with $16 \mathrm{~s}$ time resolution, using the standard PCA and HEXTE reduction steps, for this observation; they are plotted in Fig. 1.

The source is in a $\alpha$ state as defined by Belloni et al. (2000). PCA dynamical power spectra, covering the entire PCA energy range $(\sim 2-100 \mathrm{keV})$, are shown in Fig. 2 together with the PCA lightcurves.

The source presents large flux variations on short time scales $(\sim 100 \mathrm{~s})$, together with a single QPO whose frequency has a similar behavior (Fig. 2). Then around time $\sim 600 \mathrm{~s}$ (first interval), and $\sim 6000 \mathrm{~s}$ (second), a large $\sim 1000$ s dip occurs (Fig. 1). During that time, the QPO frequency varies from $9 \mathrm{~Hz}$ to $2.25 \mathrm{~Hz}$, and a strong second QPO appears with a frequency $\sim$ twice that of the fundamental, following the same frequency variations (Fig. 2). Then around relative time 2032 s (first interval), and
$7716 \mathrm{~s}$ (second interval), a sudden and large soft X-ray spike, reaching $\sim 4.8 \times($ respectively $\sim 6.4 \times$ ) the dip minimum flux, for the first (respectively second) interval, occurs and the source returns to a state similar to the one before the dip. Here the harmonic disappears, while the fundamental returns to a larger frequency and behaves as before the dip. In addition we show in Fig. 3 DPS in the five energy ranges defined in Sect. 2, together with the corresponding lightcurves. One can immediately see that above $20 \mathrm{keV}$ the harmonic is absent or very faint, and that above $40 \mathrm{keV}$ (probably due to the high noise) the QPO disappears. We also see in Fig. 3 the evolution of the flux variations with the energy; the large dip seems to be smoothed with the energy.

We extracted from the soft lightcurves the relative time and the value of the flux of the peak occuring just before the dip (relative time $554 \mathrm{~s}$, for the first interval, and 6064, for the second one); we then re-did the same procedure for the minimum of the dip (relative time $954 \mathrm{~s}$ for the first interval, and $6368 \mathrm{~s}$ for the second one), and we thus could estimate the relative amplitude of the variation of the flux, at the time where, also, the fundamental QPO sees its frequency varying from 9 to $2.25 \mathrm{~Hz}$. We did this in each energy range, at the same times (allowing a maximum of two bins ( $\sim 32 \mathrm{~s}$ ) of difference between each range). Results are shown in Table 2.

Note that the soft spike corresponds in the higher energy range (above $20 \mathrm{keV}$ ) to a sudden decrease of the flux, indicating the cooling, or the disappearance of a part of the corona (multi-wavelength results can be found in Ueda et al. 2002). 


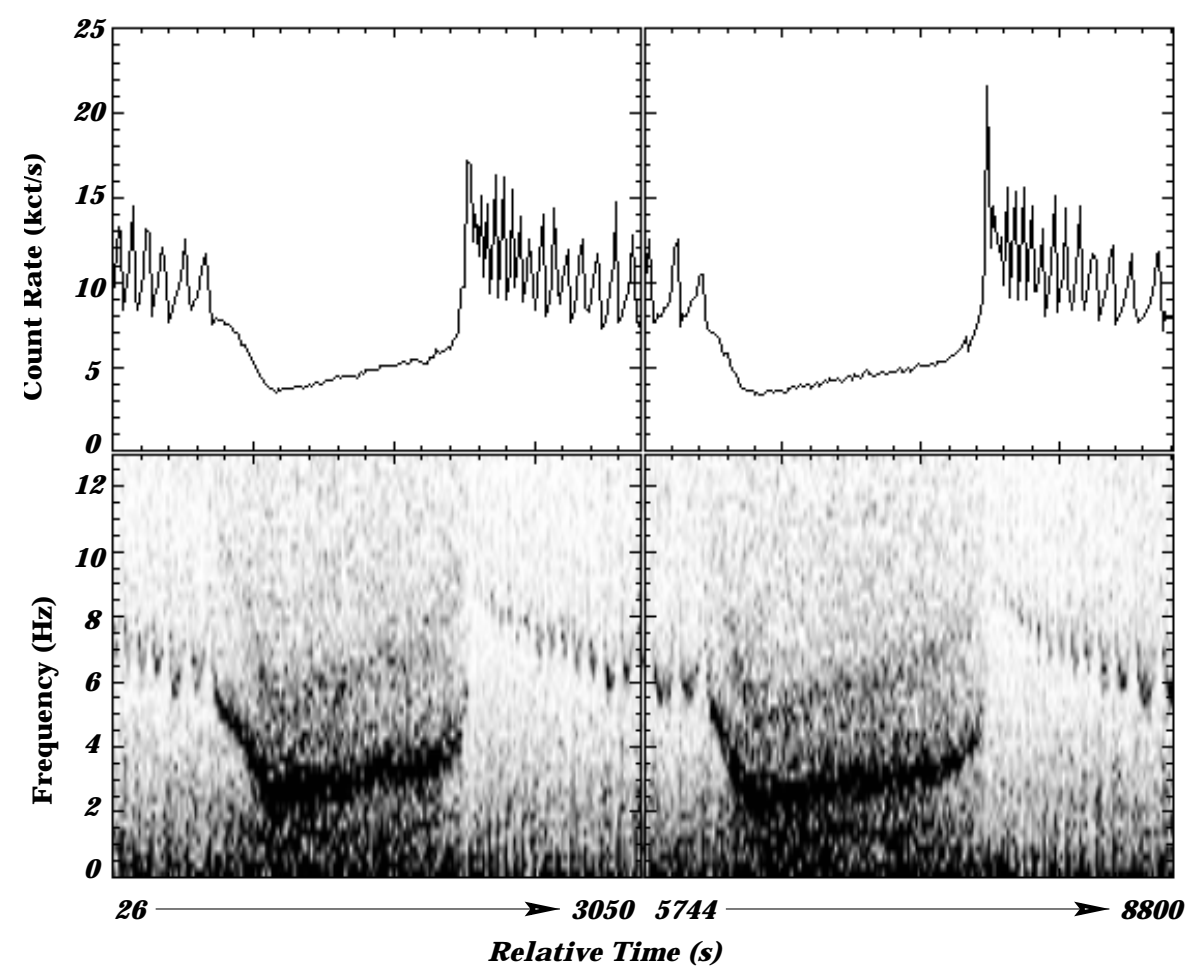

Fig. 2. PCA Lightcurve of the source during the whole observation; Lower panel: dynamical power spectra of the source on April 17th; the gap in the data corresponds to occultation due to the orbit. $X$ axis is the relative time (time 0 is defined in Table 1), in units of s. $Y$ axis is in kcts/s (upper panels), and in $\mathrm{Hz}$ (lower ones).

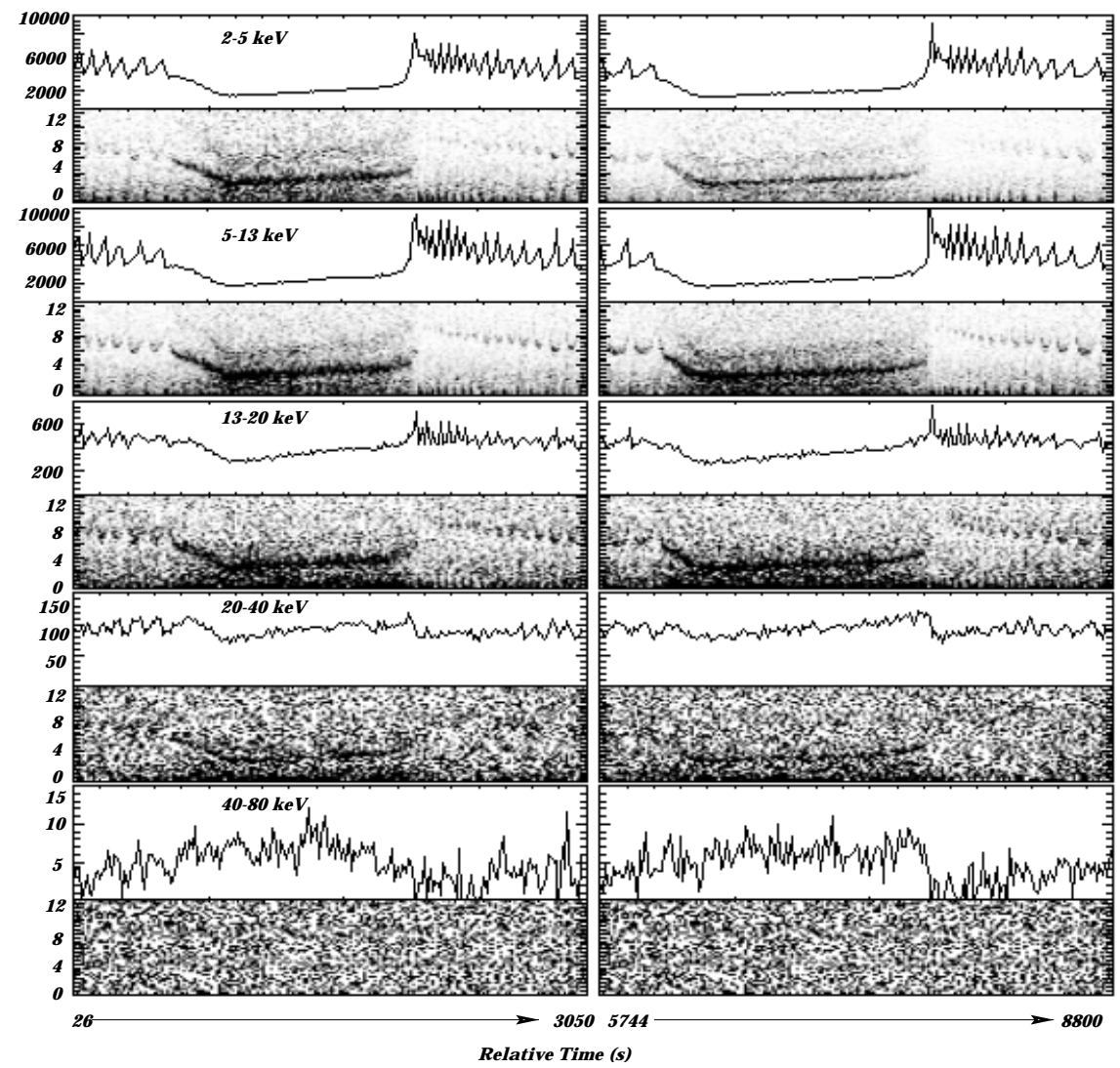

Fig. 3. Dynamical power spectra of the source on April 17th in the five PCA energy ranges; $X$ axis in all cases are the time, covering relative time from $24 \mathrm{~s}$ to $3050 \mathrm{~s}$ (Left), and relative time from $5744 \mathrm{~s}$ to $8800 \mathrm{~s}$ (Right); upper panels are the standard 2 lightcurves in the indicated energy ranges. $Y$ axis are in units of kcts/s for the upper panels, and in units of Hz for the lower ones. 
Table 2. Variations of the flux with the energy, between the last peak before the dip, and the bottom of the dip for the two intervals of April 17.

\begin{tabular}{ccc}
\hline Energy Range $(\mathrm{keV})$ & Variation Rate Interval \#1 (\%) & Variation Rate Interval \#2 (\%) \\
\hline \hline $2-5$ & $72.95 \pm 0.63$ & $71.19 \pm 0.66$ \\
\hline $5-13$ & $70.24 \pm 0.61$ & $69.61 \pm 0.63$ \\
\hline $13-20$ & $47.84 \pm 1.78$ & $42.37 \pm 1.88$ \\
\hline $20-40$ & $22.18 \pm 4.26$ & $21.29 \pm 4.07$ \\
\hline
\end{tabular}

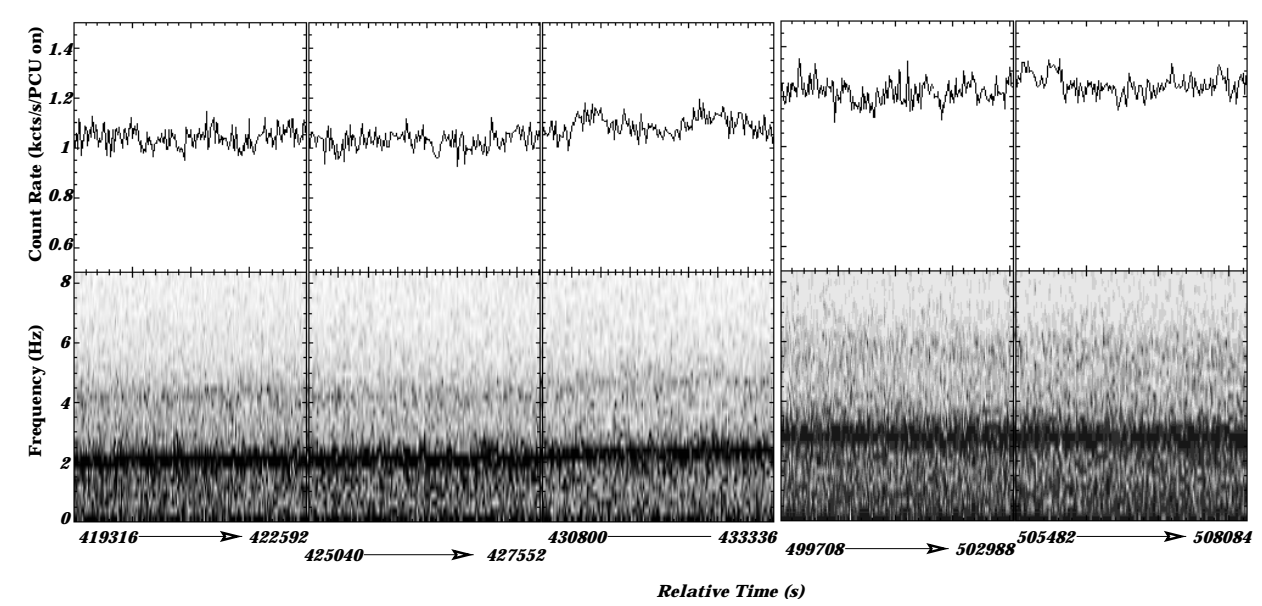

Fig. 4. Plot of the $16 \mathrm{~s}$ PCA lightcurves (upper panel), and the dynamical power spectra (bottom), covering the three good time intervals of April 22nd (left Panel) and April 23rd (Right Panel). Once again time zero is April 17th good time interval start. $Y$ axis of upper panels is in unit of kcts/s/PCUs on (see Table 1 for the PCA configuration over each interval), while that of lower panels is in $\mathrm{Hz}$.

\section{Second and third observation: April 22nd and 23rd}

As the lightcurves and dynamical power spectra did not present variations as strong as on the previous date, we did not focus here on the dynamical evolution of the QPO, but we just tried to correlate the QPO parameters with the energy range. Figure 4 shows the lightcurves with the dynamical power spectra from all the GTIs of both observations. The source is in a $\chi$ state of Belloni et al. (2000), characterized by a steady flux.

Power spectra covering the entire PCA range,shown in Fig. 5, are fitted with a model consisting of two broad lorentzians (continuum), plus sharper ones, modeling the QPO features. When the presence of the QPOs was not obvious, we estimated the parameters by freezing the Lorentzian centroid frequency to the value found in the other energy ranges, and allowing both the width and the power to vary. In the case of the $40-80 \mathrm{keV}$ range, since the statistics from single interval was poor, we choosed to merged the observations were the QPO frequency was found to be close, i.e. intervals \#1 and \#2 from April 22, and intervals \#1 and \#2 from April 23; interval \#3 from April 22 was fitted alone. Results from the fits for all the energy ranges defined in Sect. 2 are shown in Table 3. No variations similar to those of April 17 are present here; the flux remains fairly constant around a mean value $1050 \mathrm{cts} / \mathrm{s} / \mathrm{PCU}$-on, for the April 22 two first intervals, rising slowly to $\sim 1100 \mathrm{cts} / \mathrm{s} / \mathrm{PCU}-\mathrm{on}$, for the
April 22 third interval, and reaching $~ 1200 \mathrm{cts} / \mathrm{s} / \mathrm{PCU}-\mathrm{on}$, on April 23. As expected, in the same time intervals the fundamental QPO sees its frequency slowly increase with time from $\sim 2.14 \mathrm{~Hz}$ (on Apr. 22) to $\sim 2.9 \mathrm{~Hz}$ (on Apr. 23) (Fig. 4, and Table 3). The harmonic is still present during the five intervals, with a frequency varying from $\sim 4.3 \mathrm{~Hz}$ (on Apr. 22), to $\sim 5.8 \mathrm{~Hz}$ on April 23 first interval.

We plotted in Fig. 7 the evolution of the QPO power vs. energy range for the five GTIs. The upper points represent the behavior of the fundamental QPO, and the lower that of the harmonic; we can see that the power of the fundamental increases up to $40 \mathrm{keV}$, and them seems to decrease, whereas that of the harmonic seems to peak between the $5-13$ and $13-20 \mathrm{keV}$ ranges. Figure 6 represents the evolution of the QPOs width vs. their frequencies. Both distributions of points can be well fitted by lines of slopes 0.390 for the fundamental, and 0.389 for the harmonic. The zero abscissa values are found to be -0.526052 for the fundamental, and -0.932459 for the harmonic (although their physical meaning is not clear). It is clearly visible on the plot that both QPOs are tightly correlated, the width of the harmonic being $\sim$ twice that of the fundamental (resulting thus in a $Q$ value $\left(=\frac{\text { frequency }}{F W H M}\right)$ similar for both).

\section{Results and interpretation}

The April 17 observation confirms and expands the conclusion of Markwardt et al. (1999) and Muno et al. (1999), 


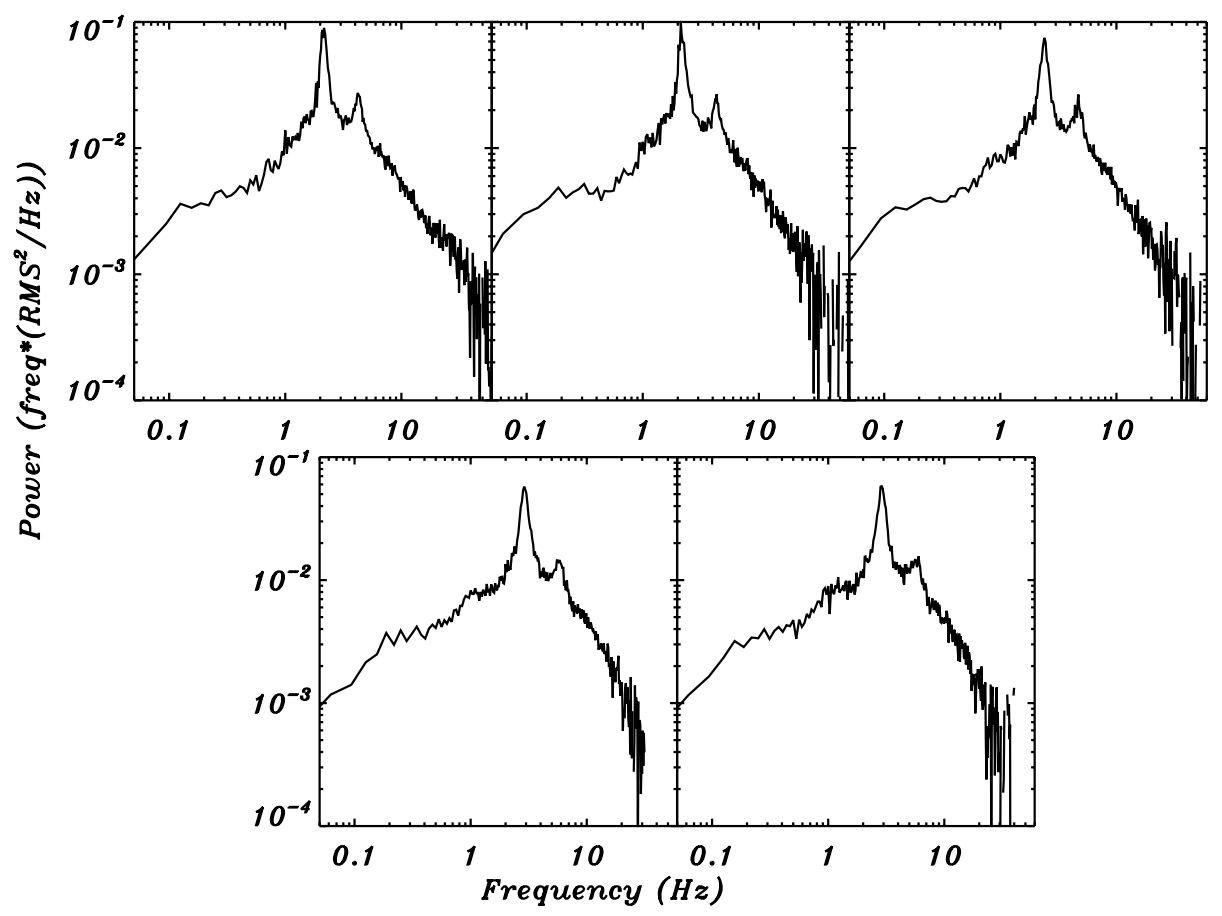

Fig. 5. Power spectra of the source on April 22nd (up), and April 23rd (lower panel). $Y$ axis is the power in terms of Freq $* \mathrm{rms}^{2} / \mathrm{Hz}$, while $X$ axis are the frequencies.

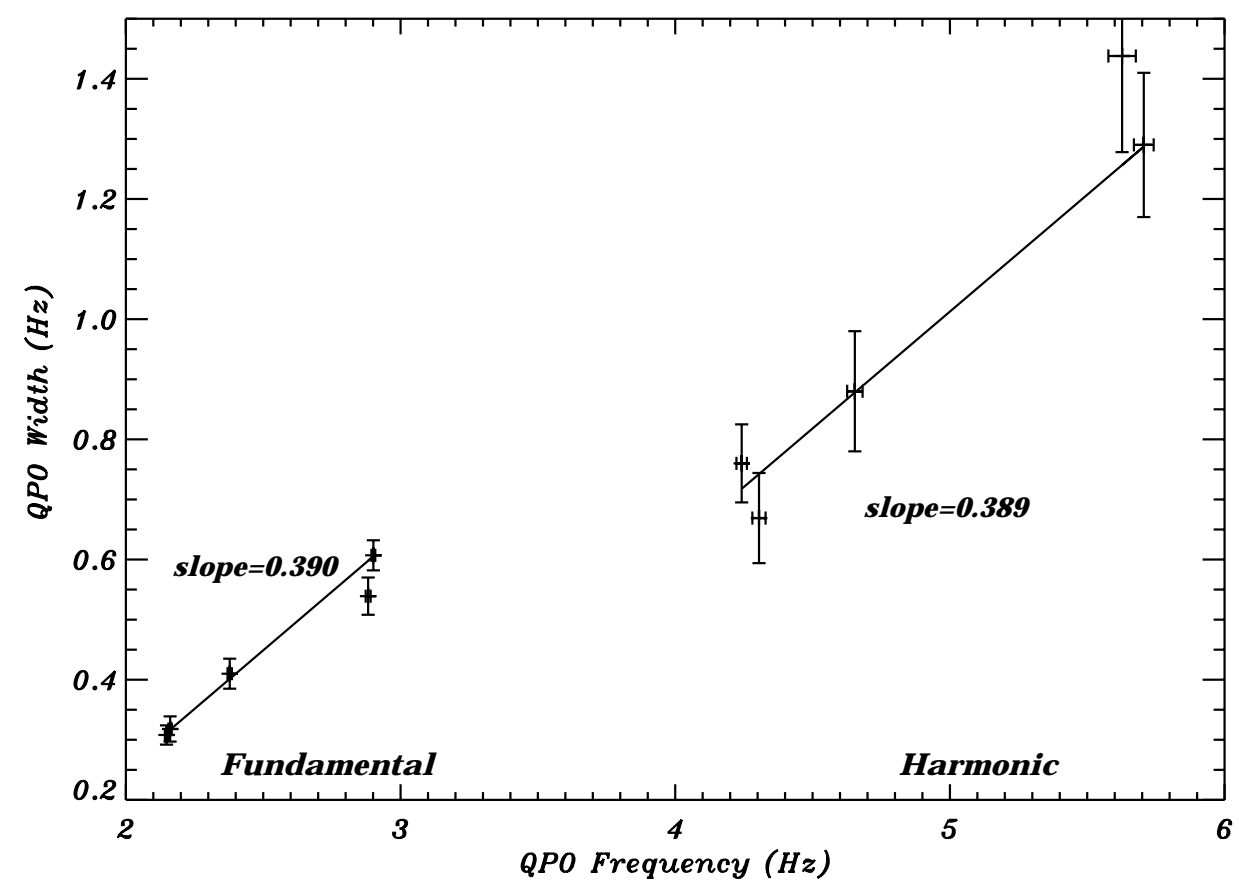

Fig. 6. Plot of QPO FWHM vs. QPO Frequency, for the five intervals covering Apr. 22nd and 23rd. Both axis are in units of $\mathrm{Hz}$. In the two case the solid lines represent the best fit. The slopes are indicated in each cases.

that the QPO frequency is better correlated with the soft flux, but seems stronger in the higher energy bands (which is confirmed by the following dates).

In addition a precise study of the lightcurve of the same date shows that the $\sim 30$ min dips are smoothed with the energy, and that the sudden increase of the soft flux (the spike) is anti correlated with the hard flux; indeed the spike, in both interval, corresponds to a major decrease of the flux in the $20-40 \mathrm{keV}$, and $40-80 \mathrm{keV}$ bands, usually considered to be emitted by the corona. The soft spike marks here the transition from the low hard state (C state of Belloni et al. 2000), to a soft high state (A-B states). Within the interpretation in terms of disk states, this transition and the rapid variations following (interpreted as rapid transitions through A B C states in Belloni et al. 2000) can be seen as a succession of rapid replenishments 
Table 3. results of the fittings for the three observations, for all the energy ranges defined in Sect. 2.

\begin{tabular}{|c|c|c|c|c|c|c|c|c|c|}
\hline Date & $\#$ & Energy range $(\mathrm{keV})$ & $f_{Q P O 1}(\mathrm{~Hz})$ & $Q_{1}$ & \%rms 1 & $f_{Q P O 2}(\mathrm{~Hz})$ & $Q_{2}$ & $\% \mathrm{rms}_{2}$ & $\chi^{2}$ (d.o.f.) \\
\hline \multirow[t]{17}{*}{04222000} & \multirow[t]{5}{*}{1} & $\mathrm{PCA}^{*}$ & $2.148_{-0.006}^{+0.006}$ & 6.97 & $12.47_{-0.57}^{+0.62}$ & $4.242_{-0.019}^{+0.019}$ & 5.58 & $6.31_{-0.42}^{+0.48}$ & $69.13(62)$ \\
\hline & & $2-5 \mathrm{keV}$ & $2.137_{-0.006}^{+0.008}$ & 7.12 & $9.92_{-0.46}^{+0.46}$ & $4.270_{-0.02}^{+0.022}$ & 5.34 & $6.26_{-0.4}^{+0.42}$ & $89.2(62)$ \\
\hline & & $5-13 \mathrm{keV}$ & $2.142_{-0.005}^{+0.007}$ & 6.88 & $14.64_{-0.61}^{+0.65}$ & $4.249_{-0.018}^{+0.019}$ & 6.85 & $6.72_{-0.46}^{+0.50}$ & $67.62(62)$ \\
\hline & & $13-20 \mathrm{keV}$ & $2.151_{-0.006}^{+0.007}$ & 6.68 & $16.16_{-0.73}^{+0.73}$ & $4.270_{-0.038}^{+0.038}$ & 7.89 & $4.63_{-0.76}^{+0.87}$ & $62.11(62)$ \\
\hline & & $20-40 \mathrm{keV}$ & $2.143_{-0.008}^{+0.01}$ & 6.00 & $16.89_{-1.03}^{+1.10}$ & 4.27 frozen & $>6.1$ & $<4.17$ & $30.19(36)$ \\
\hline & \multirow[t]{5}{*}{2} & PCA & $2.161_{-0.006}^{+0.007}$ & 6.79 & $12.27_{-0.75}^{+0.78}$ & $4.305_{-0.024}^{+0.024}$ & 6.43 & $5.39_{-0.51}^{+0.57}$ & $102.5(62)$ \\
\hline & & $2-5 \mathrm{keV}$ & $2.152_{-0.007}^{+0.008}$ & 7.24 & $9.70_{-0.66}^{+0.72}$ & $4.326_{-0.025}^{+0.025}$ & 6.21 & $5.72_{-0.52}^{+0.63}$ & $108.6(62)$ \\
\hline & & $5-13 \mathrm{keV}$ & $2.162_{-0.007}^{+0.006}$ & 6.90 & $14.53_{-0.78}^{+0.79}$ & $4.286_{-0.023}^{+0.024}$ & 7.10 & $6.24_{-0.59}^{+0.64}$ & $80.10(62)$ \\
\hline & & $13-20 \mathrm{keV}$ & $2.169_{-0.009}^{0.008}$ & 5.60 & $16.95_{-1.26}^{+1.28}$ & $4.333_{-0.092}^{+0.102}$ & 5.82 & $4.71_{-1.35}^{+1.75}$ & $87.52(42)$ \\
\hline & & $20-40 \mathrm{keV}$ & $2.182_{-0.012}^{+0.012}$ & 6.28 & $15.62_{-1.53}^{+1.66}$ & 4.30 frozen & $>6.14$ & $<4.12$ & $46.24(47)$ \\
\hline & 1-2 Merged & $40-80 \mathrm{keV}$ & 2.15 Frozen & $>15$ & $<14.21$ & & & & $7.69(10)$ \\
\hline & \multirow[t]{6}{*}{3} & $\mathrm{PCA}$ & $2.378_{-0.008}^{+0.008}$ & 5.8 & $12.31_{-0.68}^{+0.70}$ & $4.654_{-0.028}^{+0.029}$ & 5.28 & $5.94_{-0.50}^{+0.55}$ & $87.98(62)$ \\
\hline & & $2-5 \mathrm{keV}$ & $2.361_{-0.008}^{+0.009}$ & 5.84 & $9.67_{-0.5}^{+0.53}$ & $4.690_{-0.025}^{+0.026}$ & 5.39 & $6.07_{-0.43}^{+0.46}$ & $94.8(62)$ \\
\hline & & $5-13 \mathrm{keV}$ & $2.382_{-0.007}^{+0.008}$ & 5.71 & $14.78_{-0.81}^{+0.86}$ & $4.691_{-0.031}^{+0.03}$ & 5.54 & $6.95_{-0.66}^{+0.7}$ & $70.54(62)$ \\
\hline & & $13-20 \mathrm{keV}$ & $2.391_{-0.009}^{+0.009}$ & 5.97 & $15.78_{-1.16}^{+1.26}$ & $4.558_{-0.1}^{+0.127}$ & 6.16 & $4.94_{-1.42}^{+1.49}$ & $100.1(62)$ \\
\hline & & $20-40 \mathrm{keV}$ & $2.376_{-0.011}^{+0.013}$ & 5.78 & $15.83_{-1.42}^{+1.52}$ & 4.65 Frozen & $>5.8$ & $<4.48$ & $43.58(36)$ \\
\hline & & $40-80 \mathrm{keV}$ & 2.35 Frozen & $>10.21$ & $<16.08$ & & & & $19.29(28)$ \\
\hline \multirow[t]{11}{*}{04232000} & \multirow[t]{5}{*}{1} & PCA & $20.901_{-0.007}^{+0.009}$ & 4.77 & $\begin{array}{c}12.07_{-0.47}^{+0.51} \\
\end{array}$ & 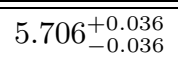 & 4.42 & $\overline{50.23_{-0.35}^{+0.43}}$ & $87.98(62)$ \\
\hline & & $2-5 \mathrm{keV}$ & $2.871_{-0.009}^{+0.009}$ & 5.31 & $9.30_{-0.44}^{+0.48}$ & $5.832_{-0.039}^{+0.041}$ & 5.12 & $4.80_{-0.41}^{+0.44}$ & $83.09(62)$ \\
\hline & & $5-13 \mathrm{keV}$ & $2.905_{-0.007}^{+0.008}$ & 5.12 & $14.14_{-0.65}^{+0.68}$ & $5.752_{-0.033}^{+0.034}$ & 5.70 & $5.71_{-0.48}^{+0.52}$ & $79.32(62)$ \\
\hline & & $13-20 \mathrm{keV}$ & $2.921_{0.009}^{+0.01}$ & 5.35 & $15.53_{-0.78}^{+0.84}$ & $5.535_{-0.146}^{+0.132}$ & 4.22 & $5.48_{-1.16}^{+1.71}$ & $122.8(62)$ \\
\hline & & $20-40 \mathrm{keV}$ & $2.925_{-0.015}^{+0.015}$ & 4.91 & $17.64_{-1.29}^{+1.38}$ & $5.940_{-0.16}^{+0.2}$ & 7.36 & $5.60_{-2.06}^{+3.10}$ & $76.89(59)$ \\
\hline & \multirow[t]{5}{*}{2} & PCA & $2.882_{-0.008}^{+0.01}$ & 5.34 & $11.47_{-0.54}^{+0.64}$ & $5.627_{-0.051}^{+0.051}$ & 3.91 & $5.41_{-0.47}^{+0.50}$ & $100.1(62)$ \\
\hline & & $2-5 \mathrm{keV}$ & $2.866_{-0.012}^{+0.012}$ & 5.83 & $8.88_{-0.57}^{+0.64}$ & $5.714_{-0.065}^{+0.067}$ & 3.19 & $6.11_{-0.71}^{+0.85}$ & $79.77(62)$ \\
\hline & & $5-13 \mathrm{keV}$ & $2.883_{-0.009}^{+0.01}$ & 5.49 & $13.86_{-0.72}^{+0.74}$ & $5.640_{-0.063}^{+0.062}$ & 3.90 & $6.33_{-0.68}^{+0.72}$ & $94.63(62)$ \\
\hline & & $13-20 \mathrm{keV}$ & $2.899_{-0.01}^{+0.013}$ & 5.71 & $14.97_{-0.87}^{+1.31}$ & 5.65 Frozen & 4.92 & $4.58_{-1.3}^{+2.16}$ & $63.84(42)$ \\
\hline & & $20-40 \mathrm{keV}$ & $2.901_{-0.019}^{+0.019}$ & 4.98 & $16.96_{-1.59}^{+1.73}$ & 5.65 Frozen & $>17.65$ & $<3.65$ & $36.86(29)$ \\
\hline & 1-2 Merged & $40-80 \mathrm{keV}$ & 2.85 Frozen & $>7.5$ & $<12.41$ & & & & $50.04(41)$ \\
\hline
\end{tabular}

* Instrument entire energy range $(\sim 2-100 \mathrm{keV})$.

and disappearances of the innermost parts of the disk (Belloni et al. 1997). The behaviour of the corona may appear difficult to understand, since the abrupt cutoff of the hard X-rays could either be the manifestation of a sudden cooling of the relativistic electrons by the re-emergence of a high soft flux, or the disappearance of the corona (by advection or ejection).

Thanks to a large number of multi-wavelength observations, the radio and infra-red behaviors of GRS 1915+105 have now been widely studied for years. In particular, former studies such as the one presented in Mirabel et al. (1998), or Eikenberry et al. (1998) had linked the soft X-ray spike (transition from low hard to soft high state) with radio and infra red flares. Dhawan et al. (2000) have shown that indeed superluminal ejections took place during abrupt change in the X-ray state of the source. More recently, Klein-Wolt et al. (2001) have found a strong correlation between radio events (radio oscillations, compact jets, large radio flares), and state $\mathrm{C}$ properties (duration, transition to other states). It is, however, to be noted that Klein-Wolt et al. did not find any simultaneous radio - alpha state observations. Furthermore, "The Largest Multi-wavelength Campaign" on GRS 1915+105 presented in Ueda et al. (2002), shows that the state transitions on Apr. 17th are followed by radio flares consistent with an ejection of material starting at the state transition. This leads us to suggest that the abrupt cutoff of the hard X-rays is more probably related to the disappearance of a part of the corona, blown away under the form of a synchrotron emitting blob of material detected in the infra red, and radio domains (Figs. 1 and 2 in Ueda et al. 2002).

On the other hand, the behavior of the QPO and its harmonic at high energies poses severe constraints on theoretical models. The decrease of the QPO power above $40 \mathrm{keV}$ may indicate that not all the corona is affected. 


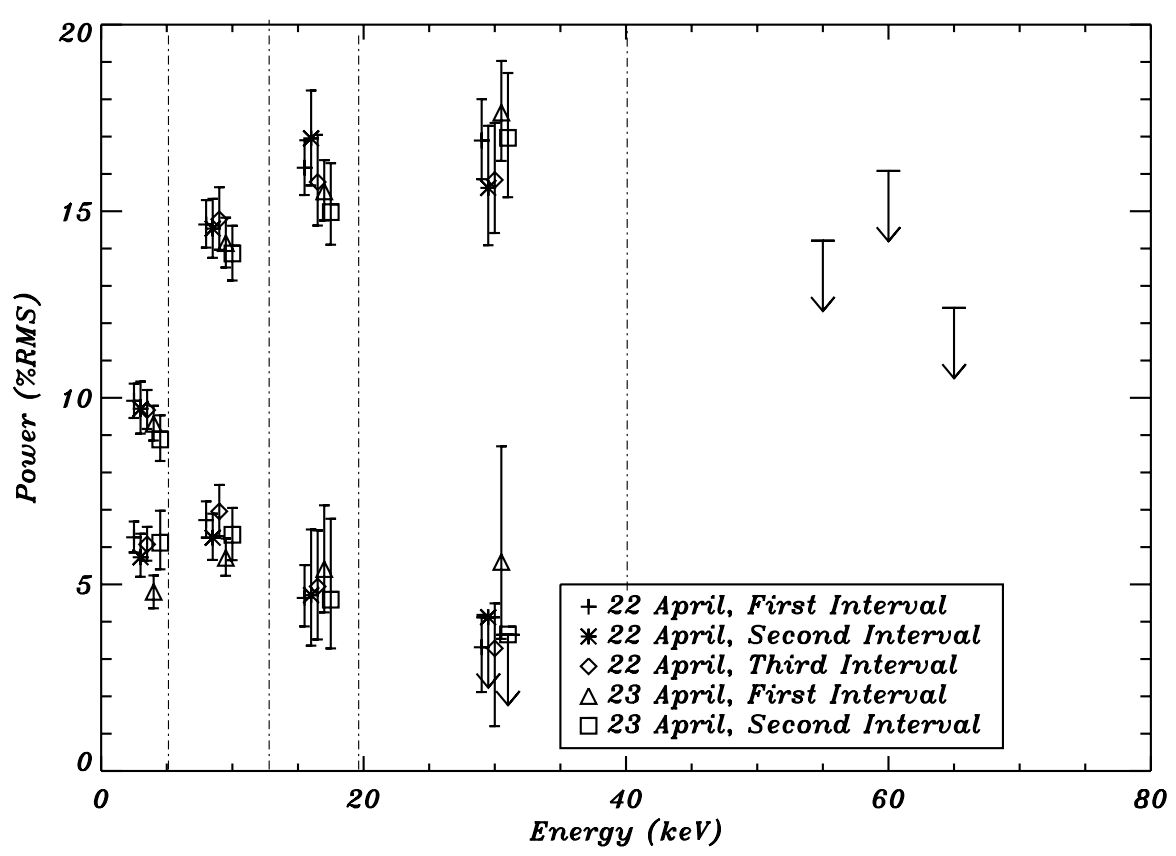

Fig. 7. Plot of the power vs. the energy range; each group of points represents the value over the whole energy range delimited by the dash-dotted line. The upper group of points in each cases corresponds to the fundamental QPO, the lower being the harmonic. Error bars are $1 \sigma$ confidence level. Arrows are the $95 \%$ upper limits. The three last arrows represent, respectively, the result from Apr. 22 intervals $1 \& 2$ merged, Apr. 22 interval 3, and Apr. 23 interval 1 \& 2 merged.

The decrease of the harmonic above $\sim 20 \mathrm{keV}$ also raises very challenging questions.

These could find an explanation in the context of the Accretion-Ejection Instability (Tagger \& Pellat 1999), which has been shown to form a rotating spiral structure in the disk, similar to galactic ones but driven by magnetic stresses rather than by self-gravity. The spiral arms should be expected to heat as well as compress the gas in the disk, and thus to appear as a rotating spiral or hot spot. The harmonic would then be a signature of the non-linear behavior of the spiral, just as the gas form shocks (and thus strong harmonics of the underlying 2armed spiral) along galactic spiral arms. The high-energy cutoff of the fundamental could, then, favor an interpretation where most or all of the quasi-periodic modulation at high energies comes, not from the comptonized corona as usually assumed, but from a hot point in the optically thick disk. This would be consistent with the previous result (Rodriguez et al. 2001; Rodriguez et al. 2002) that the anomalously small color radius of the disk, often observed in some Black-Hole Binaries, could actually be interpreted by the black-body emission of a small area hot point in the disk. We could in principle have an estimate of its physical size, by adding a blackbody model in the spectral fits (such as the BBODYRAD model of XSPEC), one of the parameters being the normalized area of the emitting region, (since the black body luminosity is proportionnal to the area). But the limited sensitivity and spectral resolution of the present data do not allow any realistic fit. We expect that future instruments will provide better constraints on this problem.
It would be very tempting to consider the width of the QPO as a measure of the size (due for example to the differential rotation acting between the inner and outer edges of the spot). But the fact that we are dealing with a QPO probably rules out this explanation, since it has to result from a quasi-stationary feature in the disk. This is precisely the case for the AEI, where a standing spiral wave results in a quasi-stationary feature rotating at a single frequency. In this context the width of the QPO would correspond to the coherence time of this pattern, fixed either by non-linear effects or by variations in the background disk equilibrium, e.g. the inner disk radius or other disk parameters (temperature, magnetization, etc.).

The spot physical properties (e.g. its temperature) may also depend on a number of external parameters, hard to deduce from the observations, such as the $\beta$ ratio (the ratio between thermal and magnetic pressure), which drives the instability (see for example Varnière et al. 2002 , for a discussion on the effects of this parameter), or even the efficiency of the instability. Indeed, in a non linear regime for example, the amount of energy deposited in the disk (under the form of shocks) would be much greater, and would locally warm it up much more than in the linear case.

Further observational and theoretical work should, however, allow to test this hypothesis: by producing, from numerical simulations of the instability (such as Caunt \& Tagger 2001), synthetic light curves of the QPO, and by fitting the observed energy dependence of the modulated light curve by a high-temperature, hotter black body over a small area of the disk rather than the usual power-law of the coronal emission. 
Acknowledgements. The authors would like to thank S. Corbel, M. Muno, P. Varnière, T. Foglizzo, and the anonymous referee for useful discussions and comments which allowed us to improve the quality of the paper.

IFM acknowledges partial support from Fundacíon Antorchas. We also thank the Athena help at GSFC for appreciable help on the RXTE data reduction processes.

This research has made use of data obtain through the High Energy Astrophysics Science Archive Center Online Service, provided by the NASA/Goddard Space Flight Center.

\section{References}

Belloni, T., Méndez, M., King, A. R., van der Klis, M., \& van Paradijs, J. 1997, ApJ, 479, L145

Belloni, T., Klein-Wolt, M., Méndez, M., van der Klis, M., \& van Paradijs, J. 2000, A\&A, 355, 271

Castro-Tirado, A., Brandt, S., Lund, N., et al. ApJS, 1994, 92, 469

Caunt, S. E., \& Tagger, M. 2001, A\&A, 367, 1095

Dhawan, V., Mirabel, I. F., \& Rodríguez, L. F. 2000, ApJ, 543, 373

Eikenberry, S. S., Matthews, K., Morgan, E. H., Remillard, R. A., \& Nelson, R. W. 1998, ApJ, 494, L61

Greiner, J., Cuby, J. G., McCaughrean, M. J., Castro-Tirado, A. J., \& Mennickent, R. E. 2001, A\&A, 373, L37

Greiner, J., Cuby, J. G., \& McCaughrean, M. J. 2001, Nature, 414, 522

Klein-Wolt, M., Fender, R. P., Pooley, G. G., et al. 2001, MNRAS, in press [astro-ph/0112044]

Markwardt, C. B., Swank, J. H., \& Taam, R. E. 1999, ApJ, 513, 37

Merloni, A., Fabian, A. C., \& Ross, R. R. 2000, MNRAS, 313, $193(\mathrm{MFR})$
Mirabel, I. F., Rodriguez, L. F., Cordier, B., Paul, J., \& Lebrun, F. 1992, Nature, 358, 215

Mirabel, I. F., \& Rodríguez, L. F. 1994, Nature, 371, 46

Mirabel, I. F., Dhawan, V., Chaty, S., et al. 1998, A\&A, 330, L9

Mirabel, I. F., \& Rodríguez, L. F. 1999, ARA\&A, 37, 409

Morgan, E. H., Remillard, R. A., \& Greiner, J. 1997, ApJ, 482, 1086

Muno, M. P., Morgan, E. H., \& Remillard, R. A. 1999, ApJ, 527,321

Psaltis, D., Belloni , T., \& van der Klis, M. 1999, ApJ, 526, 262

Rodriguez, J., Varnière, P., Tagger, M., \& Durouchoux, P. 2001, Proc. of the Third Microquasar Workshop: Granada Workshop on galactic relativistic jet sources, ed. A. J. Castro-Tirado, J. Greiner, \& J. M. Paredes, Astrophysics and Space Science, of the 3rd microquasar workshop, Granada (Spain) [astro-ph/0010635]

Rodriguez, J., Varnière, P., Tagger, M., \& Durouchoux, P. 2002, A\&A, accepted

Sobczak, G. J., McClintock, J. E., Remillard, R. A., et al. 2000, ApJ, 531, 537 (SMR)

Swank, J., Chen, X., Markwardt, C., \& Taam, R. 1997, Proc. of Conf. Accretion Processes in Astrophysics: Some Like it Hot, held at U. Md., October 1997, ed. S. Holt, \& T. Kallman

Tagger, M., \& Pellat, R. 1999, A\&A, 349, 1003 (TP99)

Tagger, M., 1999, Proc. of the 5th Compton Symp., Portshmouth (USA) [astro-ph/9910365]

Ueda, Y., Yamaoka, K., Sánchez-Fernández, C., et al. 2002, ApJ, in press

Varnière, P., \& Tagger, M. 2001, Proc. of the Gamma 2001, Symp. Baltimore MD, April 2001, ed. N. Gehrels, C. Shrader, \& S. Ritz

Varnière, P., Rodriguez, J., \& Tagger, M. 2002, A\&A, accepted 